Advances in Baking Technology 


\title{
Advances in Baking Technology
}

\author{
Edited by \\ BASIL S. KAMEL \\ ICI-Atkemix \\ Brantford \\ Ontario \\ and \\ CLYDE E. STAUFFER \\ Technical Foods Consultant \\ Cincinnati \\ Ohio
}

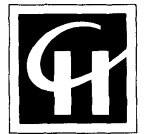

Springer-Science+Business Media, B.V. 
First edition 1993

C 1993 Springer Science+Business Media Dordrecht

Originally published by Chapman \& Hall in 1993

Softcover reprint of the hardcover 1st edition 1993

Typeset in 10/12pt Times by ROM Data Ltd, Falmouth, Cornwall

ISBN 978-0-7514-0055-7 ISBN 978-1-4899-7256-9 (eBook)

DOI 10.1007/978-1-4899-7256-9

Apart from any fair dealing for the purposes of research or private study, or criticism or review, as permitted under the UK Copyright Designs and Patents Act, 1988, this publication may not be reproduced, stored, or transmitted, in any form or by any means, without the prior permission in writing of the publishers, or in the case of reprographic reproduction only in accordance with the terms of the licences issued by the Copyright Licensing Agency in the UK, or in accordance with the terms of licences issued by the appropriate Reproduction Rights Organization outside the UK. Enquiries concerning reproduction outside the terms stated here should be sent to the publishers at the Glasgow address printed on this page.

The publisher makes no representation, express or implied, with regard to the accuracy of the information contained in this book and cannot accept any legal responsibility or liability for any errors or omissions that may be made.

A catalogue record for this book is available from the British Library 


\section{Preface}

Fundamentally, baking may seem to be a rather static topic: mix flour, water, leavening and various flavoring materials, and heat the mixture to gelatinize starch and denature the protein. However, this millenia-old process has been undergoing rapid change during this century, and even more during the last two decades. Numerous new ingredients have been developed for improving the nature of the finished baked product. New methods for heating the mixture are being used. In addition the demands of the consumer for decreased calories, increased fiber levels, decreased fat and sodium content, and more convenient products, have engendered numerous product development projects.

We have selected topics that address many of these developments in baking technology. The authors have been drawn from both Europe and North America, to reflect the fact that technology applications today are relevant on an international basis. The ingredients covered range from the most basic (wheat and rye flour) to the latest fads (dietary fiber, fat replacers). Processes include basic bread production (but with recent innovations in the plant), as well as newer methods, such as microwave baking, extrusion, and freezing of doughs and products. Those tests more useful in product development, such as rheological measurements of dough and finished baked goods, as well as sensory evaluation, are also discussed in some detail.

The topics should be of interest to anyone involved in developing baked products, in translating laboratory developments to practical production, or in ensuring the quality of ingredients purchased and the products made for sale. In short, we address the concerns of those people involved with technical and technological aspects of the bakery industry.

B.S.K.

C.E.S. 


\section{Acknowledgements}

We would like to recognize those people who have contributed to the usefulness of this book by reviewing chapters for us: Dr. John deMan, Mr. Wulf Doerry, Dr. Patrick Dreese, Dr. Elizabeth Gullett, Dr. Tony Hunt, Dr. Simon Jackel, Mr. Bill Knightly, and Dr. G. Mittal. 


\section{Contributors}

Dr M.C. Bourne

Mr J. Brown

Dr W. Bushuk

Dr J. Holas

Dr B.S. Kamel

Dr J. Kratochvil

Mr K. Kulp

Mr R.C. Miller

Professor J.G. Ponte

Dr J.Pŕíhoda

Dr V.F. Rasper

Dr M.G. Scanlon
Department of Food Science and Technology, Cornell University, Geneva, New York 14456-0462, USA

European Process Plant Ltd. Correspondence to 59 Lawers Crescent, Polmont, Falkirk FK2 0RQ

Grain Industry Research Group, Department of Food Science, c/o St Paul's College, University of Manitoba, Winnipeg, MB, R3T 2N2, Canada

SEDBA Ltd, Jankovcova 18, 17037 Prague 7, Czechoslovakia

Atkemix Inc (ICI Specialty Chemicals), PO Box 1085, Brantford, Ontario N3T 5T2, Canada

Flour Milling and Baking Research Association, Chorleywood, Herts, WD3 5SH, UK

2230 Grandview Terrace, Manhattan, Kansas 66502, USA

R.D.2, Box 413, Auburn, New York 13021, USA

Kansas State University, Manhattan, Kansas 66506, USA

Institute of Chemical Technology, Department of Carbohydrate Chemistry and Technology, Technická 5, 16628 Praha 6, Czechoslovakia

Department of Food Science, University of Guelph, Guelph, Ontario N1G 2W1, Canada

Department of Food Science, University of Manitoba, Winnipeg, MB, R3T 2N2, Canada 
Dr C.S. Setser

Mr R. Silva

Mr R.F. Schiffmann

Dr C.E. Stauffer
Department of Foods and Nutrition, Kansas State University, Manhattan, Kansas 66506, USA

Imperial Holly Corporation, Sugarland, Texas 774870009, USA

R.F. Schiffmann Associates Inc, 149 West 88 Street, New York 10024, USA

631 Christopal Drive, Cincinnati, Ohio 45231, USA 


\section{Contents}

1 Wheat and wheat flours 1

W. BUSKUK and M.G. SCANLON

1.1 Introduction $\quad 1$

1.2 Wheat classification 2

1.3 World wheats 3

1.3.1 Production 3

1.3.2 Morphology and composition 4

$\begin{array}{lll}\text { 1.3.3 Utilization } & 6\end{array}$

$\begin{array}{lll}1.3 .4 & \text { World wheats } & 7\end{array}$

$\begin{array}{lll}1.4 & \text { Wheat milling } & 10\end{array}$

1.4.1 Hard wheat milling 10

$\begin{array}{ll}\text { 1.4.2 Soft wheat milling } & 12\end{array}$

1.4.3 Automation and optimization in milling 13

$\begin{array}{lll}1.5 & \text { Wheat flours } & 14\end{array}$

1.5.1 Flours for bread and similar products 14

1.5.2 Flours for pastry, cookies and cakes 15

$\begin{array}{ll}\text { 1.5.3 Flours around the world } & 15\end{array}$

$\begin{array}{lll}1.6 & \text { Conclusions } & 18\end{array}$

$\begin{array}{ll}\text { References } & 18\end{array}$

2 Rye flour, wholemeal breads and rye breads $\quad 20$

J. PŘíHODA, J. HOLAS and J. KRATOCHVÍL

$2.1 \quad$ World rye production 20

2.2 Technological aspects of rye flour composition and component properties 22

2.3 Nutritional aspects of rye production and consumption 25

$\begin{array}{ll}2.4 & \text { Methods of rye quality assessment } \\ 2.5 & 27\end{array}$

$\begin{array}{ll}2.5 & \text { Milling of rye } \\ 2.6 & 29\end{array}$

2.6 Baking technology of rye products $\quad 30$

References $\quad 36$ 
J. BROWN

3.1 Introduction 38

3.1.1 Ingredients 38

3.1.2 Dough mixing and development 39

3.1.3 Processing and baking equipment 39

3.2 Basic facts on breadmaking 39

3.2.1 General information 39

3.3 Breadmaking processes $\quad 42$

3.3.1 Introduction 42

3.3.2 Bread type and quality 42

3.3.3 Dough development (maturing) process 42

3.4 Bulk fermentation processes 43

3.4.1 Process parameters 43

3.4.2 Forms of bulk fermentation process 44

3.5 Mechanical development processes 47

3.5.1 US systems 47

3.5.2 The Chorleywood Bread Process 48

3.6 Activated dough development process 54

3.6.1 Introduction 54

3.6.2 Main features of Activated Dough Development 55

3.7 Spiral mixing for no-time doughs with ascorbic acid 56

3.7.1 Origin of process 56

3.7.2 Reasons for the popularity of spiral mixers 57

3.7.3 Spiral mixing process recipes 58

3.7.4 Australian no-time dough process 58

3.8 Potassium bromate - the effects of its ban in the UK 59

3.9 Further breadmaking processes 60

3.9.1 American sponge and dough $\quad 60$

3.9.2 Brews 60

3.9.3 The green dough process $\quad 60$

3.9.4 Standard Dutch bread 61

3.9.5 Dr Calvel's autolysis process $\quad 62$

3.9.6 German sour dough process 62

3.10 Ingredients $\quad 63$

3.10 .1 Flour 63

3.10.2 Dried gluten 64

3.10.3 Emulsifiers $\quad 64$

3.10 .4 Soya flour $\quad 66$

3.10 .5 Fats $\quad 66$

3.10.6 Sugar $\quad 66$

$\begin{array}{ll}3.10 .7 & \text { Malt flour and fungal } \alpha \text {-amylase }\end{array}$ 
3.10.8 Compound dough additives (also called bread improvers and dough conditioners) $\quad 68$

$\begin{array}{lll}3.10 .9 & \text { Yeast } & 71\end{array}$

3.11 Processing equipment $\quad 72$

3.11.1 Chorleywood Bread Process 72

3.11.2 Spiral mixers 73

3.11.3 Dough handling plant $\quad 74$

3.11.4 Moulding/panning methods $\quad 75$

3.11.5 Final proof 75

$\begin{array}{lll}3.11 .6 & \text { Proof time } & 77\end{array}$

3.11.7 Retarding and retarder proving 77

3.11.8 Dough handling prior to baking 80

3.11.9 Steaming $\quad 81$

3.11 .10 Baking $\quad 81$

3.11.11 Bread cooling and packaging 83

3.12 Developments in retail baking $\quad 84$

3.12.1 Instore baking $\quad 84$

3.12.2 Bake-off units 85

$\begin{array}{ll}\text { 3.12.3 Part-baked products for retail sale } & 87\end{array}$

$\begin{array}{ll}\text { References } & 87\end{array}$

4 Frozen dough production $\quad 88$

C.E. STAUFFER

$\begin{array}{lll}4.1 & \text { Introduction } & 88\end{array}$

4.2 Ingredients, formulation $\quad 89$

$\begin{array}{llr}4.2 .1 & \text { Flour } & 89\end{array}$

$\begin{array}{lll}4.2 .2 & \text { Yeast } & 90\end{array}$

4.2.3 Emulsifiers 91

$\begin{array}{lll}4.2 .4 & \text { Oxidants } & 92\end{array}$

4.2.5 Other ingredients 92

4.3 Dough processing 93

4.3.1 Mixing 93

4.3.2 Makeup 96

4.3.3 Chemically leavened products 97

$\begin{array}{lll}4.4 & \text { Freezing and storage } & 98\end{array}$

$\begin{array}{llr}\text { 4.4.1 Equipment } & 98\end{array}$

4.4.2 Freezing doughs 101

$\begin{array}{lll}4.4 .3 & \text { Storage } & 102\end{array}$

4.5 Use in the bakery 103

4.5.1 Thawing and proofing 103

4.5.2 Baking and finishing 105

References 105 
V.F. RASPER

$\begin{array}{lll}5.1 & \text { Introduction } & 107\end{array}$

$\begin{array}{ll}5.2 & \text { Fundamental dough rheology } \\ & 112\end{array}$

5.3 Dough and gluten rheology at elevated temperatures 117

$\begin{array}{lll}5.4 & \text { Rheology of other flour systems } & 123\end{array}$

$\begin{array}{lll}5.5 & \text { Rheology of spongy systems } & 126\end{array}$

$\begin{array}{lll}5.6 & \text { Concluding remarks } & 128\end{array}$

$\begin{array}{ll}\text { References } & 129\end{array}$

6 Texture measurements on finished baked goods 134

M.C. BOURNE

$\begin{array}{lll}6.1 & \text { Introduction } & 134\end{array}$

$\begin{array}{ll}6.2 \text { Deformation } & 134\end{array}$

$\begin{array}{lll}6.3 & \text { Snapping } & 139\end{array}$

$\begin{array}{lll}6.4 & \text { Puncture } & 140\end{array}$

6.5 BBIRA biscuit texture meter 143

$\begin{array}{lll}6.6 & \text { Volume } & 143\end{array}$

$\begin{array}{lll}6.7 & \text { Texture profile analysis (TPA) } & 144\end{array}$

$\begin{array}{lll}6.8 & \text { Texture press } & 148\end{array}$

$\begin{array}{lll}6.9 & \text { Other tests } & 148\end{array}$

References 149

$7 \quad$ Enzymes as dough improvers $\quad 152$

K. KULP

$\begin{array}{llr}7.1 & \text { Introduction } & 152\end{array}$

7.1.1 General improving effects on doughs 152

7.1.2 Characteristics of commercial enzyme preparations 152

7.1.3 Improvements of baked products 153

7.1.4 Flour components altered by enzymes 153

$\begin{array}{lll}7.2 & \text { Amylases } & 153\end{array}$

7.2.1 $\alpha$-Amylase $\quad 154$

$\begin{array}{ll}7.2 .2 \text { Glucoamylases } & 155\end{array}$

7.2.3 General properties of amylases $\quad 157$

7.2.4 Individual improving functions of amylases 160

$\begin{array}{lll}7.3 & \text { Proteolytic enzymes } & 166\end{array}$

7.3.1 Modification of doughs by proteases 168

7.3.2 Functional effects of proteolytic enzymes 169

$\begin{array}{lll}7.4 & \text { Pentosanases and cellulases } & 169\end{array}$

7.4.1 Functionality of cellulases and pentosanases $\quad 170$ 
7.4.2 Functionality of pentosanases in cookie and cracker manufacturing

7.5 Lipoxygenase

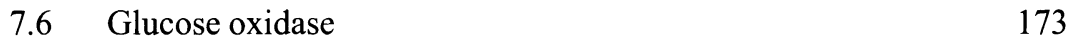

$\begin{array}{lll}7.7 & \text { Sulfhydryl oxidase } & 173\end{array}$

7.8 Enzyme-based replacers of bromate 174

$\begin{array}{lll}7.9 & \text { Summary } & 176\end{array}$

$\begin{array}{ll}\text { References } & 176\end{array}$

8 Emulsifiers in baking 179

B.S. KAMEL and J.G. PONTE JR

$\begin{array}{lll}8.1 \quad \text { Introduction } & 179\end{array}$

8.2 Classification and regulation 183

8.2.1 Classification 184

8.2.2 Regulation 185

8.3 Synthetic food emulsifiers 186

8.3.1 Mono- and diglycerides 186

8.3.2 Mono- and diglyceride derivatives 188

8.4 Function of surfactant in baked products 195

8.5 Mechanism of surfactant function in different baked goods 199

8.5.1 Emulsification and foam stabilization 199

8.5.2 Starch interaction 202

8.5.3 Protein interaction 204

8.5.4 Physical states of emulsifiers in water 205

8.6 Staling of bakery foods 206

8.6.1 Theories of staling 206

8.6.2 Theories of bread staling 207

8.6.3 Cake staling 211

8.6.4 Measurement of staling 211

8.7 Concluding remarks 216

References 217

$\begin{array}{ll}\text { Further reading } & 221\end{array}$

9 Lecithin and phospholipids in baked goods 223

R. SILVA

9.1 Introduction 223

9.2 Review of lecithin chemistry 227

9.2.1 Lecithin the natural emulsifier 230

9.2.2 Lecithin in baking 232

9.2.3 Lecithin in breadmaking 233

9.2.4 Lecithin in cakes 235 
9.2.5 Lecithin in doughnuts 235

9.2.6 Lecithin in cookies 236

9.2.7 Lecithin as a release agent 236

9.2.8 Lecithin as an anti-oxidant 237

9.3 Miscellaneous bakery applications $\quad 238$

9.3.1 Bakery coatings 238

9.3.2 Dry mixes 238

9.3.3 Lecithin - regulatory aspects 238

9.3.4 Lecithin - specifications 239

9.4 Lecithin - chemical modification 241

9.5 Lecithin - enzyme modification 242

9.6 Lecithin - fractionation 244

9.7 Final comment 251

$\begin{array}{ll}\text { References } & 251\end{array}$

10 Sensory evaluation $\quad 254$

C.S. SETSER

10.1 Sensory properties of bakery products 254

10.1.1 Introduction 254

$\begin{array}{lll}10.1 .2 & \text { Flavor } & 254\end{array}$

10.1.3 Texture 256

$\begin{array}{ll}10.1 .4 \text { Appearance } & 257\end{array}$

$\begin{array}{ll}10.2 \text { Methodology } & 258\end{array}$

10.2.1 Test categories $\quad 259$

10.2.2 Mechanics and panel operations 272

$\begin{array}{lll}10.3 & \text { Statistics for sensory studies } & 277\end{array}$

$\begin{array}{lll}\text { 10.3.1 Experimental design } & 277\end{array}$

10.3.2 Data analysis and interpretation 278

10.3.3 Relationships of sensory data to instrumental measures 280

10.3.4 Validity checks for sensory studies 281

10.4 Conclusion $\quad 285$

Appendix $\quad 286$

$\begin{array}{ll}\text { References } & 287\end{array}$

11 Microwave technology in baking 292

R.F. SCHIFFMANN

$\begin{array}{lll}11.1 & \text { Introduction } & 292\end{array}$

11.2 Process advantage of microwave systems 293

11.2.1 Advantages of microwave heating 293

11.2.2 Criteria for the selection of a microwave
processing system

11.3 Microwave heating fundamentals 295 
11.3.1 Electromagnetic waves 295

11.3.2 Heating mechanism 297

11.3.3 Interaction of microwave fields with materials 298

11.4 Equipment for microwave processing 302

11.4.1 The power supply and generator 302

$\begin{array}{ll}\text { 11.4.2 Applicators } & 302\end{array}$

11.4.3 Control systems 303

11.4.4 Economics of microwave processing systems 303

11.5 Microwave systems for industrial processing in the baking industry 304

11.5.1 Baking 304

11.5.2 Proofing 308

11.5.3 Pasteurization 308

11.5.4 Thawing 309

11.6 Radio frequency (RF) baking 310

11.7 Microwave ovens 310

11.7.1 Characteristics of microwave ovens 311

11.7.2 Microwavable baked products 311

11.8 The future of microwave baking in industry 313

References $\quad 313$

12 Extrusion of baked products 316

R.C. MILLER

12.1 Elements of extrusion processing 316

12.2 Principles of extrusion 318

12.2.1 Screw extrusion 318

12.2.2 Lamella (roller) and piston (ram) extruders $\quad 321$

12.2.3 Operating lines 322

12.3 Extrusion processes in baking operations 322

12.4 Baking and extrusion cooking 325

12.5 Some fundamental principles 326

12.5.1 Laminar flow and shear 326

12.5.2 Product uniformity and residence time distribution 328

12.6 To extrude or to bake - a further comparison 329

12.7 Conclusions 334

References $\quad 334$

13 Fats and fat replacers 336

C.E. STAUFFER

13.1 Introduction 336

13.2 Natural fats 337

13.2.1 Chemical structure 337

13.2.2 Extraction and refining 342 
13.3 Bakery shortenings 344

13.3.1 Traditional shortenings 344

13.3.2 Tailored fats 345

13.3.3 Shortening properties $\quad 352$

13.4 Shortening functionality 356

13.4.1 Bread, rolls 356

13.4.2 Danish, puff pastry 357

13.4.3 Cakes, muffins 358

$\begin{array}{ll}13.4 .4 \text { Biscuits } & 359\end{array}$

13.4.5 Crackers 362

13.4.6 Deep-fried snacks, doughnuts 363

13.5 Nutritional implications 364

13.5.1 Obesity 364

13.5.2 Serum cholesterol and cardiovascular disease $\quad 364$

13.5.3 Prostaglandins 366

13.6 Fat replacement in baked foods 367

13.6.1 Low and non-caloric lipids 367

13.6.2 Protein-based replacers 368

13.6.3 Carbohydrate-based replacers 368

13.6.4 Process changes 369

$\begin{array}{ll}\text { References } & 369\end{array}$

14 Dietary fiber, analysis, physiology and calorie reduction 371

C.E. STAUFFER

$\begin{array}{lll}14.1 & \text { Introduction } & 371\end{array}$

14.2 Structure of dietary fiber 372

14.2.1 Insoluble dietary fibers $\quad 373$

14.2.2 Soluble dietary fibers 377

14.2.3 Analytical methods $\quad 381$

14.2.4 Dietary fiber sources 383

14.3 Applications of fiber in baked goods 384

14.3.1 Water absorption and retention 385

14.3.2 Bread - high fiber and reduced calorie 386

14.3.3 Cakes, muffins 391

14.3.4 Biscuits, crackers 392

14.4 Physiological effects of dietary fiber 393

14.4.1 Weight reduction 393

14.4.2 Control of diabetes 393

14.4.3 Cancer reduction 395

14.4.4 Decrease in blood cholesterol 396

14.4.5 Physical gastrointestinal tract disorders 396

14.4.6 Some adverse effects of fiber 397

References 397

Index 399 